\title{
Haul Truck Assisted Driving Technologies Based on the Atmospheric Degradation Physical Models
}

\author{
Enji Sun ${ }^{1,2} a^{*}$, Xingkai Zhang ${ }^{1, b}$ \\ ${ }^{1}$ China Academy of Safety Science and Technology, Beijing, China, 100012 \\ 2 Virginia Polytechnic Institute and State University, VA, United States \\ aenjisun@gmail.com, bzhangxk@chinasafety.ac.cn,
}

Keywords: surface mining, haul truck, atmospheric degradation physical models, environment detection

\begin{abstract}
Haul truck transportation is one of the most important transportation methods in large surface mining operations. It is a key factor that affects the mining productivity and cost effective, however, its accidents rate and dangers are far higher than other types of developing transportation in the surface mining accidents. For the environmental characteristics of surface mining roads and the weather factors, this paper eliminates the open-pit haul truck security risks during haul trucks transportation by the installation of the haul trucks CCD camera equipment. This paper proposes haul trucks transportation environment detection video display enhancement technologies based on the physical models of atmospheric degradation, recovery algorithm to improve the haul trucks drivers' visual distance under the rain, snow, fog dust, etc. lower visibility severe weather conditions in surface mining operations. The results indicate that this technology can increase reliability and reduce uncertainty in surface mining operations.
\end{abstract}

\section{Introduction}

According to the statistics of Mine Safety and Health Administration, there are quite accidents involved with haul trucks in surface mining. Between the year of 1990 and 2014, there are almost 1800 fatal accidents attributed to the general category of, among which a total of 163 fatalities or 20.4 percent were haul truck-related. ${ }^{[1,2]}$ Generally speaking, when huge equipment moving to the edges of the slope or dumping spots, there 198 accidents occurred each year, there of them are fatalities: ${ }^{[3,4]}$ About 3000 workers died in all kinds of accidents in the mining fields, mining safety is in a serious condition in China. ${ }^{[5]}$ Haul trucks involved accidents are the major type accident in the surface mining. According to AnTaiBao surface mining accidents statics, in the past decades, haul truck involved accidents is more than $75 \%$, which causes serious damages and life lost. ${ }^{[6]}$ HeiDaiGou surface mining located in Zhungeer Qi, Ordos City, the Inner Mongolia. In the past 17 years, there are transportations accidents occurred every year. Proportion of the haul trucks accidents is more than $70 \%{ }^{[7]}$

Till now, most researches focus on the haul truck's fleet management system. ${ }^{[8-10]}$ There have been several studies analyzing truck-related fatalities. ${ }^{[11-13]}$ Haul trucks assisted driving system has been designed and used to improve the drive safety. ${ }^{[14]}$ Also, some researches provided the haul truck driver fatigue detection methods by using PERCLOS methods. Due to the wide blind spots block the driver's view, especially under the fog, storm, blizzard and heavy dust conditions. ${ }^{[15]}$ The visibility of transportation environment system becomes poor and resulted in the drivers visual judgments distance is limited and short. ${ }^{[16]}$ As almost $90 \%$ of the road information is coming from driver's eyes. The limitation of driver's view increases the safety risks for the haul trucks, surrounding vehicles and pedestrians. ${ }^{[17]}$

In this condition, the main objective of this research was to build upon previous studies and designed environment detection video display enhancement technologies based on the atmospheric physical degradation models for haul trucks transportation. The recovery algorithm was applied to improve the haul truck driver's visual distance under lower visibility severe weather conditions such 
as the rain, snow, fog, dust, etc. in surface mining operations. This technology can provide assisted driving information to the drivers daily, especially in the fog, dust or dim light conditions. It can reduce the safety risk of haul truck's drivers due to the effects of blinds spots and severe environment conditions in the surface mining.

\section{The environment detection for haul trucks}

\section{Atmospheric degradation physical models recovery algorithm}

The difficulty of the atmospheric model for the detection and recovery of the road transport environment is to acquire the depth of monitoring image. If the depth of image can be analyzed in advance, it will be easy for the recovery of atmospheric model. In most cases, the degraded images are practical applications of the scene. The reality of scene is real, in another word, it is measurable in the reality. While the scene itself is fixed. It can be restored by using the depth of the image restoration no matter how the weather changes, how the image is degraded. For the same scene, if the degradation images can be get under two different weather conditions, this method does not consider the scattering coefficients of two degraded images. The calculated depth is a proportion relation with the actual depth of the images. As the atmospheric degradation model is:

$$
E=I_{\infty} \rho e^{-\beta d}+I_{\infty}\left(1-e^{-\beta d}\right)
$$

Where $E$ is the brightness of degraded images, $I_{\infty}$ is the sky brightness of the degraded images, $\rho$ is normalized radiation rate. The calculation of normalized radiation rate for the same scene is:

$$
\rho^{i}=1-\sum_{j}\left(1-\rho^{i}\right) \frac{I_{\infty}-E^{i}}{\sum_{i}\left(I_{\infty}-E^{i}\right)} .
$$

In Eq.2, the normalized radiation rate of all points of the same depth is not known. In this way, each point should be given an estimation of an initial value, then these values are on iteration until each point reaches balance in Eq.2. The iteration results are the normalized radiation rate of all points of this image depth. The same calculation is carried out on the other depth regions until the normalized radiation rates of all points are solved. And then the brightness of the sky is multiplied by normalized radiation rate. The final results are the restored images of the environment detection in the surface mining. The advantage of this method is that the scattering coefficient can be ignored and the brightness of the sky can be calculated automatically while the image depth is calculated.

\section{Parameter estimation of recovery algorithm}

1) The estimation of the brightness of the sky $E_{\infty}$

Firstly, the image should be divided into the sky section $\left(\Omega_{S}\right)$ and non-sky section $\left(\Omega_{B}\right)$, and make the scene as $\Omega=\Omega_{S} \cup \Omega_{B}$, then the brightness of sky $E_{\infty}$ is the average greyness of sky section, then:

$E_{\infty}=\frac{1}{\mathrm{~N}_{S}} \sum_{n_{i} \in \Omega_{S}} I n_{i}$

2) The estimation of atmospheric scattering coefficient $\beta$

Atmospheric scattering coefficient $\beta$ can be calculated by $\beta=3.912 / R_{v}$, where $R_{v}$ is the 
visible distance under current condition.

3) The estimation of distance from the view spot to receive device $d$

A scene vanishing point is selected on the border region of far horizon and sky connection in open-pit mine road transportation environment detection image. A series of concentric circles are made by using this vanishing point as the center of a circle. The spots on the same circumference can be approximated to have the same scene depth, so that:

$$
d=d_{\text {min }}+k\left(d_{\text {max }}-d_{\text {min }}\right), \quad 0 \leq k \leq 1 .
$$

$d_{\max }$ and $d_{\min }$ are the farthest and nearest distance respectively, $k$ is determined by the ratio of the corresponding point to the farthest point and the nearest point. $\alpha \in[0,1]$ is the distance from the vanishing point to the nearest spot. Because of the depth of the scenic spot is not linear with the degree of degradation of the weather, the value is closer to infinite, the depth will suddenly increase, and the weather degradation is more serious usually. So we construct a monotonically decreasing concave function on the interval section in $[0,1]$. As $d$ increases as the decrease of $\alpha$, if $k=1-\alpha^{n / 2}$ $(0<n<1)$, then:

$$
d=d_{\text {min }}+\left(1-\alpha^{n / 2}\right)\left(d_{\max }-d_{\text {min }}\right) .
$$

From the atmospheric scattering model, it can be seen that the brightness of the field is formed by the brightness of the object's surface reflected light and the brightness of the light produced from the atmosphere. In a sunny day, the reflection of the object surface is the main function, so the effect of the light from the atmosphere can be removed by removing the light from the atmosphere. By using image data estimation basic information, the scattering coefficient $\beta$ increases as the density increases. The value of $\beta$ can be evaluated according to degradation images. For maximum visibility is $50 \mathrm{~m}$ under fog condition, the value of $\beta$ is about $78 \mathrm{~km}^{-1}$. The value of $\beta$ would be around 0.4 $\mathrm{km}^{-1}$ if the maximum visibility is in $10 \mathrm{~km}$. Because of the different density of weather, the weather interference is different, so the image is restored by changing the value of $\beta$ according to the weather impaction. The recovery of atmospheric degradation physical models and the improvements of the visibility of the road environment detection of truck drivers and distance is shown in Fig. 1.

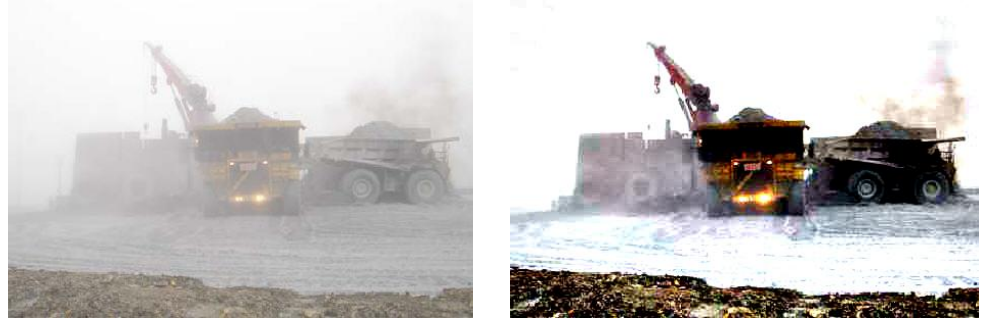

Fig. 1. The image recovery and enhancement under dust condition for haul trucks

\section{Summary}

In this paper, CCD cameras, which are installed in the haul trucks in surface mining, eliminate the safety hidden trouble of the truck's blind spots, and realize the visual distance of truck drivers in the rain, snow, fog, dust and so on. By using the real-time visual image enhancement technology, transportation road scene image are captured and to judge the sharpness of the image using multi sensor information fusion to enhance the image for the driver in a severe environment. It provides good vision and improves open-pit mine haul trucks transport safety. Assisted monitoring image is 
the direct assisted driving technology, but it can also lead to a new risk as it attracted the driver's attention. It is necessary to continue to study the development of a variety of monitoring technology for open pit haul truck safety monitoring and early warning technology.

\section{Acknowledgment}

This work was supported by National Natural Science Foundation of China (71373245, 50974109), the fundamental research funding of China Academy of Safety Science and Technology (2013JBKY03, 2014JBKY04, 2015JBKY06), and National Key Technology R\&D Program of the Ministry of Science and Technology (2013BAK04B03).

\section{References}

[1] Enji Sun, Antonio Nieto, Zhongxue Li, Vladislav Kecojevic. An Integrated Information Technology Assisted Driving System to Improve Mine Trucks-Related Safety. Safety Science, 2010, 48(10):1490-1497.

[2] Enji Sun, Antonio Nieto, Zhongxue Li. GPS and Google Earth based 3D assisted driving system for trucks in surface mines. Mining Science and Technology, 2010, 20(1):138-142.

[3] Nieto A, Dagdelen K. Development of a Dump Edge and Vehicle Proximity Warning System Based on GPS and Wireless Networks to Improve Safety in Open Pit Mines. SME Transactions, 2006, 3(20):11-20.

[4] Mine Safety and Health Administration MSHA. MSHA Fatality Statistics, 2014[EB/OL]. http://www.msha.gov/stats/charts/chartshome.html

[5] Qiming Wang, Aimin Zhou. The control of geological disasters in mining engineering Mining Technology, 2004, 4(4): 20-23.

[6] Erfu Zhao, Minghai Duan. Analysis and control of trucks transport accidents in surface mine. Opencast coal mining technology, 2007, (3): 53-55.

[7] Weintraub A.,Barros L.,Magendzo A.,Ibarra F.,Ortiz C. A truck dispatching system for a large open pit mine. Operational Research '87. Proceedings of the Eleventh International Conference,1988, 650-652.

[8] Chong Chen, Ying Zhang, Qiang Wang, Wenli Xu, Yanfeng Wang. Simulation of Truck Dispatching System in Surface Mine Based on Extend. Journal of System Simulation, 2007, 19(4): 914-921.

[9] GAO Yunbo, ZHAO Yunsheng, CUI wei. Simulation study on vehicle anti-collision model of Highway. Journal of Safety Science and Techno logy, 2009, 5(4):47-50.

[10] Xiaoyu Sun, Xifu Wang. The manufacture mobile vehicle-mounted terminal using in open-pit truck automation dispatching system. Journal of China Coal Society, 2008, 3(8): 876-880.

[11] Kecojevic, V., Komljenovic, D., Groves, W., Radomsky, M., 2007. An analysis of equipmentrelated fatal accidents in U.S. mining operations: 1995-2005, Safety Science 45, 864-874.

[12] Kecojevic, V., Md-Nor, Z.A., 2009. Hazard identification for equipment-related fatal incidents in the U.S. underground coal mining. Journal of Coal Science and Engineering 12 (1), 1-6.

[13] Ruff, T., Coleman, P. \& Martini, L., 2011, Machine-related injuries in the US mining industry and priorities for safety research. International Journal of Injury Control \& Safety Promotion, 18(1), $11-20$. 
[14] Antonio Nieto, Enji Sun, ZhongXue Li. Real-time assisted driving in open pit mining operations using Google Earth. Mining engineering, 2010, 62(2): 21-24.

[15] Sun Enji, Zhang Xingkai, Li Zhongxue, Wang Yunhai. Haul trucks 3D assisted driving and proximity warning system in mines. Metal Mine, 2012, (12): 102-109,122.

[16] Enji Sun, Xingkai Zhang. 3D Assisted driving system for haul trucks in surface mining. Transportation, Mechanical, and Electrical Engineering (TMEE), 2011 International Conference on, 2011:363- 366.

[17] Enji SUN, Antonio NIETO, Zhongxue LI. GPS and Google Earth based 3D assisted driving system for trucks in surface mines. Mining Science and Technology, 2010, 20(1): 138-142.

[18] Enji Sun,Xingkai Zhang, Zongxue Li, Yunhai Wang. The machine vision based surface mining drive fatigue monitoring. Journal of Liaoning Technical University (Natural Science), 2012, 31(1):21-25. 